\title{
Letter
}

\section{Reversed Currents in Charged Liquid Bridges}

\author{
Klaus Morawetz ${ }^{1,2,3}$ \\ 1 Münster University of Applied Sciences, Stegerwaldstrasse 39, 48565 Steinfurt, Germany; \\ morawetz@fh-muenster.de; Tel.: +49-25-5196-2411 \\ 2 International Institute of Physics (IIP), Av. Odilon Gomes de Lima 1722, 59078-400 Natal, Brazil \\ 3 Max-Planck-Institute for the Physics of Complex Systems, 01187 Dresden, Germany
}

Academic Editors: Elmar Christof Fuchs, Jakob Woisetschläger, Adam D. Wexler and Astrid H. Paulitsch-Fuchs Received: 24 March 2017; Accepted: 15 May 2017; Published: 17 May 2017

\begin{abstract}
The velocity profile in a water bridge is reanalyzed. Assuming hypothetically that the bulk charge has a radial distribution, a surface potential is formed that is analogous to the Zeta potential. The Navier-Stokes equation is solved, neglecting the convective term; then, analytically and for special field and potential ranges, a sign change of the total mass flow is reported caused by the radial charge distribution.
\end{abstract}

Keywords: classical transport; electrokinetic effects; electrohydrodynamics; electrorheological fluids; Navier-Stokes equations

PACS: 05.60.Cd; 47.57.jd;47.65.-d; 83.80.Gv

\section{Introduction}

Although it has been known for over 100 years [1], the formation of a water bridge between two beakers under high voltage has not lost its fascination. It is interesting to understand the underlying electrohydrodynamics of such water bridges at nanoscale since liquid bridging is not restricted to water but can also be observed in other liquids [2] and is characteristic of polar dielectric liquids [3]. Therefore, its cause might be in electrohydrodynamics [4] rather than in molecular-specific structures.

Applications can be found in atomic force microscopy [5]; phenomena in micro-fluidics [6]; up to electrowetting problems [7] to confine chemical reactions [8]. Molecular dynamical simulations [9] help to understand the underlying mechanism, especially what leads a water filament to overcome the surface tension [10]. The formation of a water bridge across the lipid bilayer is considered as the first stage of electroporation for the intrusion of individual water molecules into the membrane interior [11]. The dynamics of charged liquids is also used for capillary jets [12]. Some current applications include ink printers and electrosprays [13,14] and the breakup dynamics of free surfaces and flows [15,16].

Such water bridges can even be stretched and used for flexible nanofluids [17]. Water bridges exhibit viscoelastic behavior with the possibility to measure its Young modulus [18]. There is an interplay between field-induced polarization, surface tension, and condensation [10,19] such that the forces of dielectric and surface tensions simultaneously hold the bridge against gravity [20]. For an overview of the different forces occurring in microelectrode structures, see [21].

In the absence of bulk charges, the forces on the water stream are caused by the pressure of the polarizability of water due to the high dielectric susceptibility $\epsilon$. This pressure leads to the catenary form of the water bridge-like a hanging chain [22]. While the simplified model of [2], employing a capacitor picture, already leads to a critical field strength for the formation of the water bridge, the catenary model [22] or a charged catenary [23] allows the determination of the static and dynamical stability conditions where charged liquid bridges are possible. The creeping height, the bridge radius and length as well as the shape of the bridge were calculated, showing an asymmetric profile in 
agreement with observations $[24,25]$. The flow profile was obtained from the Navier-Stokes equation leading to a mean velocity which combines charge transport with neutral mass flow [26]. It shows that even the uncharged catenary provides a minimal critical field strength for the water bridge formation in dependence on the length of the bridge. This critical field strength is modified if charges are present in the bridge [23]. The occurrence of charges due to dissociation has also been used to explain the I-V hysteresis reported in [27].

In this paper, it is assumed that the charge carriers play an important role, such that surface charges might be formed. This hypothetical assumption is analyzed and leads to a surface potential which essentially influences the charge transport. Since both mass and charge transport are coupled, here we find the possibility that the mass transport might show a reverse behavior dependent on the parameters. This study is motivated by the bidirectional flow visualized recently in [28] and measured with mass and charge transfer in [29].

\section{Velocity Profile with Bulk Charges}

The link between the Ohm picture of a constant current density of charged particles and the dependence of the mass flow on the cross-area for incompressible fluids has been found in a combined flow expression [26]. Possible charges with density $\rho_{c}$ in water move according to the applied electric field $E$ and will create a mean mass motion of density $\rho$. Such access charge has been found in $[30,31]$. Let us first recall the main steps for such a transport picture and then investigate how a radial charge distribution changes the results.

In the stationary case and neglecting the nonlinear convection, the Navier-Stokes equation [32] reads

$$
\eta \nabla^{2} \vec{v}-\nabla P+\rho_{c} \vec{E}=0
$$

with the gradient of the electric pressure given in the direction of the bridge of length $L$ by

$$
-\nabla P=\frac{\epsilon_{0}(\epsilon-1) E^{2}}{2 L}=\frac{b(E)}{2 L} \rho g
$$

with the dielectric constant $\epsilon$ and introducing the creeping height

$$
b(E)=\frac{\epsilon_{0}(\epsilon-1) E^{2}}{\rho g} .
$$

For details about the gradient of the electric pressure, see Equations (8) and (22) of [26]. This leads from (1) to the equation for the radial-dependent velocity

$$
\frac{\eta}{\rho g} \frac{d}{d r}\left(r \frac{d v}{d r}\right)+r\left(\frac{b}{2 L}+c\right)=0
$$

where we have introduced the dimensionless ratio $c$ of the force density on the charges by the field, $\rho_{c} E$, to the gravitational force density, $\rho g$, in the form

$$
c\left(\rho_{c}, E\right)=\frac{\rho_{c} E}{\rho g} .
$$

The solution of (4) provides the velocity profile in the direction of the bridge as

$$
v(r)-v(R)=v_{0}\left(\frac{b}{2 L}+c\right)\left(1-\frac{r^{2}}{R^{2}}\right)
$$


where $R$ is the radius of the bridge and the characteristic velocity reads

$$
v_{0}=\frac{\rho g R^{2}}{4 \eta} .
$$

In [26], it was assumed that the undetermined velocity at the surface of the bridge $v(R)$ vanishes.

\section{Surface Potential}

The direction of the flux not only depends on the applied electrostatic field but also on the relative electronegativity of the electrodes [33]. A complex bi-directional mass transfer pattern has been found in such bridges $[3,28]$. Especially in the latter paper, the bidirectional flow was observed by charged isotopes. The light water flows above the heavier water. Thus, it is justified to assume bulk charges. Already in [4], it was considered that the bulk charges might be realized in a surface sheet. Ionized charges at the anode migrate to the outer surface of the bridge [30]. Therefore, the migration of charges to the surface should be considered to be forming a charged surface sheet which will be an extension of [26]. Now, let us develop step by step the idea of a surface charge and the corresponding potential.

The force on an ionic charge $e_{i}$ in an electric field with small velocities $v_{i}$ can be well approximated by the Stokes force

$$
\vec{F}=e_{i} \vec{E}=6 \pi \eta r_{i} \vec{v}_{i}
$$

where $r_{i}$ is the radius and $\eta$ the viscosity. With the conductivity $\sigma$, the $\zeta$ potential can be defined from the velocity

$$
\vec{v}_{i}=\frac{e_{i}}{6 \pi \eta r_{i}} \vec{E}=\frac{\sigma}{\rho_{i}} \vec{E}=-\epsilon \epsilon_{0} \frac{\zeta}{\eta} \vec{E}
$$

and can be written in two forms

$$
\zeta=-\frac{e_{i}}{6 \pi \epsilon \epsilon_{0} r_{i}}=-\frac{\sigma \eta}{\epsilon \epsilon_{0} \rho_{i}} .
$$

This $\zeta$ potential actually describes the electric potential at the surface of the bridge which we will now see from the analogous idea as one describes electro-osmose. We assume that the charge density in the bridge consists of a homogeneous bulk charge $\rho_{b}$,

$$
\rho_{c}=\rho_{b}+\rho_{r}(r),
$$

and a radial-dependent modulation of the charge density $\rho_{r}(r)$ according to a distribution that is analogous to the screening cloud. This is a purely hypothetical assumption which we will justify by the observation that it leads exactly to the finite potential $\zeta$ at the surface. In other words, in the case that the surface potential of the bridge is at the boundary between liquid and air, the following considerations are the result. The Poisson equation for the electrostatic potential $\Psi$ in that case reads

$$
\nabla^{2} \Psi=-\frac{\rho_{r}}{\epsilon \epsilon_{0}}=-\frac{1}{\epsilon \epsilon_{0}} \sum_{i} n_{i} e_{i}\left(\mathrm{e}^{-e_{i} \Psi / T}-1\right) \approx \kappa^{2} \Psi
$$

with the squared inverse screening length $\kappa^{2}=\sum_{i} \frac{n_{i} e_{i}^{2}}{\epsilon \epsilon_{0} T}$. Here, we have assumed that the integrated spatial inhomogeneous charge density modulation is zero such that besides the homogeneous $\rho_{b}$ no total access charge remains. The Poisson Equation (12) is readily solved for a radial dependence

$$
\Psi(r)=\zeta \frac{I_{0}(\kappa r)}{I_{0}(\kappa R)}
$$


with the potential at the surface $\zeta=\Psi(R)$ and the Bessel function $I_{0}$. From (12), we obtain the radial dependence of the spatial modulation of the charge density

$$
\rho_{r}(r)=-\epsilon \epsilon_{0} \nabla^{2} \Psi=-\epsilon \epsilon_{0} \kappa^{2} \Psi(r)
$$

from which the additional body force follows

$$
\vec{f}(r)=\rho_{r}(r) \vec{E}=-\epsilon \epsilon_{0} \kappa^{2} \zeta \frac{I_{0}(\kappa r)}{I_{0}(\kappa R)} \vec{E} .
$$

\section{Velocity Profile with Bulk and Surface Charges}

Now we are ready to solve the Navier-Stokes Equation (1) including this additional force density (15)

$$
\eta \Delta \vec{v}-\vec{\nabla} P+\vec{f}+\rho_{b} \vec{E}=0 .
$$

The bridge extends in the $x$-direction with the following geometry

$$
\vec{E}=(E, 0,0): \quad \vec{v}=(v(r), 0,0)
$$

as well as a constant pressure gradient $\nabla P=\partial_{x} P$ of (2) This leads to a simple equation for the radial dependence that is analogous to (4)

$$
\frac{\eta}{r} \frac{d}{d r}\left(r \frac{d v}{d r}\right)+\rho g\left(\frac{b}{2 L}+c\right)=\epsilon \epsilon_{0} \kappa^{2} \zeta E \frac{I_{0}(\kappa r)}{I_{0}(\kappa R)} .
$$

Demanding that the solution is finite at $r=0$, one can integrate (18) and instead of (6), we obtain

$$
\frac{v(r)-v(R)}{v_{0}}=\left(\frac{b}{2 L}+c\right)\left(1-\frac{r^{2}}{R^{2}}\right)+\frac{4 \epsilon \epsilon_{0} \zeta E}{\rho g R^{2}}\left[\frac{I_{0}(\kappa r)}{I_{0}(\kappa R)}-1\right] .
$$

This gives the velocity profile in dependence on the radius if a surface potential $\zeta$ is present. The first part without such potential (6), has been obtained in [26] considering the bulk charges and the dielectric pressure due to the electric field. The second term is the new contribution arising from the surface charge.

The total volume flow relative to the surface flow can be calculated from (19) as

$$
J(R)=2 \pi \int_{0}^{R}[v(r)-v(R)] r d r=J_{0}\left[(\kappa R)^{2}-8 \frac{\zeta}{\zeta_{0}} \frac{I_{2}(\kappa R)}{I_{0}(\kappa R)}\right]
$$

with

$$
\begin{aligned}
J_{0} & =\frac{\pi v_{0}}{2 \kappa^{2}}\left(\frac{b}{2 L}+c\right) \\
\zeta_{0} & =\left(\frac{b}{2 L}+c\right) \frac{\rho g}{\epsilon \epsilon_{0} E \kappa^{2}}=\frac{(\epsilon-1) E}{2 \epsilon \kappa^{2} L}+\frac{\rho_{b}}{\epsilon \epsilon_{0} \kappa^{2}} .
\end{aligned}
$$

The result is presented in Figure 1 and one can see that the flow can change the direction if the $\zeta$ potential exceeds a critical value. This value can be found by observing in Figure 1 that the sign change appears for small $\kappa R$ leading to a zero in $J(R)$ at $(\kappa R)_{0}$. Therefore, from an expansion of (20) for $\kappa R$, the sign change can appear for

$$
\zeta>\zeta_{0}
$$


with (10) and (21). The zero nonlinear $(\kappa R)_{0}$ in dependence on the $\zeta$ potential is plotted in Figure 2 . The region of inverse flow is given for values of $\kappa R$ which are smaller than $(\kappa R)_{0}$ indicated by the shaded area.

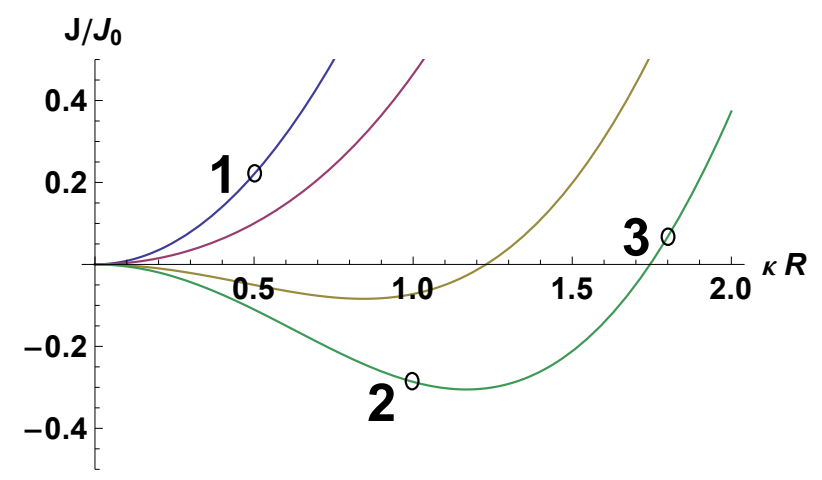

Figure 1. The total mass flow (20) vs. the dimensionless radius of the bridge. The parameter $8 \zeta / \zeta_{0}=1,5,10,12$ from left to right. The numbered points represent the regions for which the velocity profile is given in Figure 3.

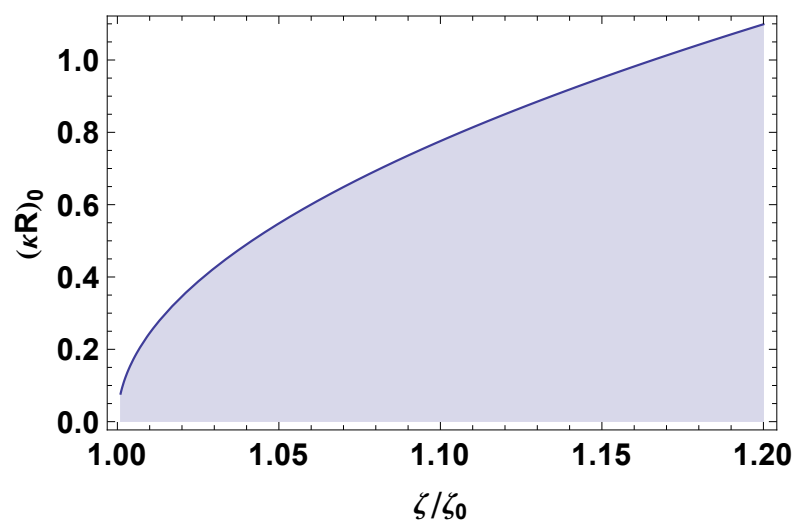

Figure 2. The critical parameter $(\kappa R)_{0}$ where the mass flow changes the sign vs. the $\zeta$ potential. The shaded area is the region where the flow reverses the sign.

With the help of (21), the radial velocity profile (19) can be recast into the form

$$
v(r)-v(R)=\frac{2 J_{0}}{\pi R^{2}}\left\{(\kappa R)^{2}\left(1-\frac{r^{2}}{R^{2}}\right)+4 \frac{\zeta}{\zeta_{0}}\left[\frac{I_{0}(\kappa r)}{I_{0}(\kappa R)}-1\right]\right\}
$$

and is presented in Figure 3. We plot the radial velocity profile of the three numbered points in Figure 1. One sees that point 3 of Figure 1 leads to a sign change in the profile such that the total mass flow has an opposite sign. Of course, this is only the ideal case when the velocity at the surface vanishes $v(R)=0$. Otherwise, we have to add the value $\pi R^{2} v(R)$ to the mass flow (20), which we assume to be small. 


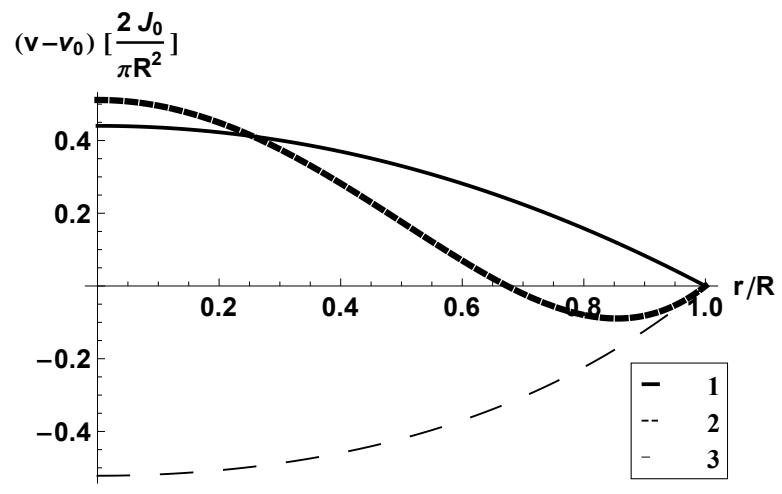

Figure 3. The radial velocity profile for the three numbered points of Figure 1.

\section{Summary}

As motivated by the recent visualizations of bidirectional flow [28], we have considered, in addition to bulk charges, a spatial modulation of the radial charge distribution such that a surface potential occurs. Solving the Navier-Stokes equation leads to a modified mass flow through the bridge. Dependent on the parameters, one can find a range where the flow is changing its direction. The observation here is that a surface potential can cause a line of zero velocity, i.e., a stagnation line of mass flow, and can lead to a sign reversal of the total mass current. One will most probably observe bi-directional mass transfer patterns. The surface potential can be thought of as being created by polar water molecules and charges separated from neutral air. In order to keep the total charge density constant, such a surface potential could balance the access charges. This is so far a purely hypothetical scenario.

Conflicts of Interest: The authors declare no conflict of interest.

\section{References}

1. Armstrong, W.G. The Electrical Engineer; The Newcastle Literary and Philosophical Society: New Castle, UK, 1893; pp. 154-155.

2. Saija, F.; Aliotta, F.; Fontanella, M.E.; Pochylski, M.; Salvato, G.; Vasi, C.; Ponterio, R.C. Communication: An extended model of liquid bridging. J. Chem. Phys. 2010, 133, 081104.

3. Wexler, A.; López Sàenz, M.; Schreer, O.; Woisetschläger, J.; Fuchs, E. The Preparation of Electrohydrodynamic Bridges from Polar Dielectric Liquids. J. Vis. Exp. 2014, 91, e51819.

4. Melcher, J.R.; Taylor, G.I. Electrohydrodynamics: A review of role of interfacial shear stresses. Ann. Rev. Fluid Mech. 1969, 1, 111-146.

5. Sacha, G.M.; Verdaguer, A.; Salmeron, M. Induced water condensation and bridge formation by electric fields in atomic force microscopy. J. Phys. Chem. B 2006, 110, 14870-14873.

6. Squires, T.M.; Quake, S.R. Microfluidics: Fluid physics at the nanoliter scale. Rev. Mod. Phys. 2005, 77, 977-1026.

7. Oh, J.M.; Ko, S.H.; Kang, K.H. Analysis of electrowetting-driven spreading of a drop in air. Phys. Fluids 2010, 22, 032002.

8. Garcia-Martin, A.; Garcia, R. Formation of nanoscale liquid menisci in electric fields. Appl. Phys. Lett. 2006, $88,123115$.

9. Chen, S.; Huang, X.; van der Vegt, N.F.A.; Wen, W.; Sheng, P. Giant Electrorheological Effect: A Microscopic Mechanism. Phys. Rev. Lett. 2010, 105, 046001.

10. Cramer, T.; Zerbetto, F.; Garcia, R. Molecular mechanism of water bridge buildup: Field-induced formation of nanoscale menisci. Langmuir 2008, 24, 6116-6120.

11. Ho, M.C.; Levine, Z.A.; Vernier, P.T. Nanoscale, Electric Field-Driven Water Bridges in Vacuum Gaps and Lipid Bilayers. J. Membr. Biol. 2013, 246, 793-801. 
12. Ganancalvo, A.M. On the theory of electrohydrodynamically driven capillary jets. J. Fluid Mech. 1997, 335, 165-188.

13. Gamero-Castano, M. Energy dissipation in electrosprays and the geometric scaling of the transition region of cone-jets. J. Fluid Mech. 2010, 662, 493-513.

14. Higuera, F. Electrodispersion of a liquid of finite electrical conductivity in an immiscible dielectric liquid. Phys. Fluids 2010, 22, 112107.

15. Eggers, J. Universal pinching of 3d axisymmetrical free-surface flow. Phys. Rev. Lett. 1993, 71, 3458-3460.

16. Eggers, J. Nonlinear dynamics and breakup of free-surface flows. Rev. Mod. Phys. 1997, 69, 865-929.

17. Chen, J.; Wang, C.; Wei, N.; Wan, R.; Gao, Y. 3D flexible water channel: Stretchability of nanoscale water bridge. Nanoscale 2016, 8, 5676-5681.

18. Teschke, O.; Soares, D.M.; Filho, J.F.V. Floating liquid bridge tensile behavior: Electric-field-induced Young's modulus measurements. Appl. Phys. Lett. 2013, 103, 251608.

19. Gómez-Monivas, S.; Sáenz, J.J.; Calleja, M.; García, R. Field-Induced Formation of Nanometer-Sized Water Bridges. Phys. Rev. Lett. 2003, 91, 056101.

20. Montazeri Namin, R.; Azizpour Lindi, S.; Amjadi, A.; Jafari, N.; Irajizad, P. Experimental investigation of the stability of the floating water bridge. Phys. Rev. E 2013, 88, 033019.

21. Ramos, A.; Morgan, H.; Green, N.G.; Castellanos, A. Ac electrokinetics: a review of forces in microelectrode structures. J. Phys. D Appl. Phys. 1998, 31, 2338-2353.

22. Widom, A.; Swain, J.; Silverberg, J.; Sivasubramanian, S.; Srivastava, Y.N. Theory of the Maxwell pressure tensor and the tension in a water bridge. Phys. Rev. E 2009, 80, 016301.

23. Morawetz, K. The effect of electromagnetic fields on a charged catenary. AIP Adv. 2012, 2, 022146.

24. Woisetschlager, J.; Gatterer, K.; Fuchs, E.C. Experiments in a floating water bridge. Exp. Fluids 2010, 48, 121-131.

25. Marin, A.G.; Lohse, D. Building water bridges in air: Electrohydrodynamics of the floating water bridge. Phys. Fluids 2010, 22, 122104.

26. Morawetz, K. Theory of water and charged liquid bridges. Phys. Rev. E 2012, 86, 026302. errata: Phys. Rev. E 86,069904 .

27. Oshurko, V.B.; Fedorov, A.N.; Ropyanoi, A.A.; Fedosov, M.V. Hysteresis and negative differential resistance of the I-V characteristic of a water bridge. Tech. Phys. 2014, 59, 1245-1248.

28. Wexler, A.; Drusová, S.; Fuchs, E.; Woisetschläger, J.; Reiter, G.; Fuchsjäger, M.; Reiter, U. Magnetic resonance imaging of flow and mass transfer in electrohydrodynamic liquid bridges. J. Vis. Exp. 2017, 20, 97.

29. Fuchs, E.C.; Agostinho, L.L.F.; Eisenhut, M.; Woisetschlager, J. Mass and charge transfer within a floating water bridge. In Laser Applications in Life Sciences; Kinnunen, M., Myllylä, R., Eds.; Proc. SPIE: Oulu, Finland, 2010; Volume 7376, p. 73761E.

30. Teschke, O.; Soares, D.M.; Gomes, W.E.; Filho, J.F.V. Floating liquid bridge charge dynamics. Phys. Fluids 2016, 28, 012105.

31. Fuchs, E.C.; Sammer, M.; Wexler, A.D.; Kuntke, P.; Woisetschläger, J. A floating water bridge produces water with excess charge. J. Phys. D Appl. Phys. 2016, 49, 125502.

32. Chandrasekharaiah, D.S. Continuum Mechanics; Academic Press: Boston, MA, USA, 1994.

33. Giuliani, L.; D’Emilia, E.; Lisi, A.; Grimaldi, S.; Brizhik, L.; Giudice, E.D. Copper ion fluxes through the floating water bridge under strong electric potential. Electromagn. Biol. Med. 2015, 34, 167-169.

(C) 2017 by the author. Licensee MDPI, Basel, Switzerland. This article is an open access article distributed under the terms and conditions of the Creative Commons Attribution (CC BY) license (http:/ / creativecommons.org/licenses/by/4.0/). 